\title{
Zusammenhänge verstehen, individuelle Unterschiede beachten und Weiterbildung hinterfragen
}

\section{Blickfang Pathogenese FOKUS \\ Systemischer Lupus erythematodes}

Endogene und Umgebungsfaktoren beeinflussen die Immunreaktion und das Anfallen apoptotischen Materials. Auf apoptotischen Zellen sind SLE-Autoantigene für das Immunsystem erkennbar. Autoantigen-Peptide werden durch Antigen-präsentierende Zellen, im Verlauf der Immunreaktion aber auch durch B-Zellen für deren spezifisches Antigen, den T-Zellen präsentiert. Notwendig ist dafür neben MHC, Peptid und T-Zell-Rezeptor (TCR) auch die Kostimulation, z.B. über CD40 und CD40-Liganden (CD40L). Mit TZell-Hilfe proliferieren B-Zellen und produzieren spezifische monoklonale Autoantikörper. Ein Teil der B-Zellen wird zu langlebigen Plasmazellen, die über Jahrzehnte Antikörper produzieren. Mit Antigen ergeben sie Immunkomplexe, die sich in Organen ablagern. Immunkomplexe aktivieren Komplement und werden von Monozyten/Makrophagen gebunden. In Folge produzieren diese Zytokine wie Tumor-Nekrose-Faktor (TNF) und Interleukin-6 (IL-6) und verursachen eine Entzündung in den Organen. Sie erzeugen aber auch das B-Zell-Zytokin BLyS/BAFF, das B-Zellen im Sinne einer Rückkopplungsschleife fördert. BLyS/BAFF wird durch Belimumab blockiert. Plasmazytoide Dendritische Zellen (pDC) reagieren auf Immunkomplexe durch die Produktion von Interferon-a (IFNa). Interferone fördern die Aktivierung Antigen-präsentierender Zellen und anderer Immunzellen. Das wird durch Blockierung des gemeinsamen Typ-I-Interferon-Rezeptors (IFNR) durch Anifrolumab gestoppt. B-Zellen werden durch die Anti-CD20-Antikörper Rituximab oder Obinutuzumab depletiert. Klinische Studien mit Antikörpern gegen CD40 (Iscalimab) und CD40L (Dapirolizumab) laufen aktuell.

Autor: Prof. Dr. Martin Aringer, Medizinische Klinik und Poliklinik III, Universitätsklinikum Carl Gustav Carus der TU Dresden

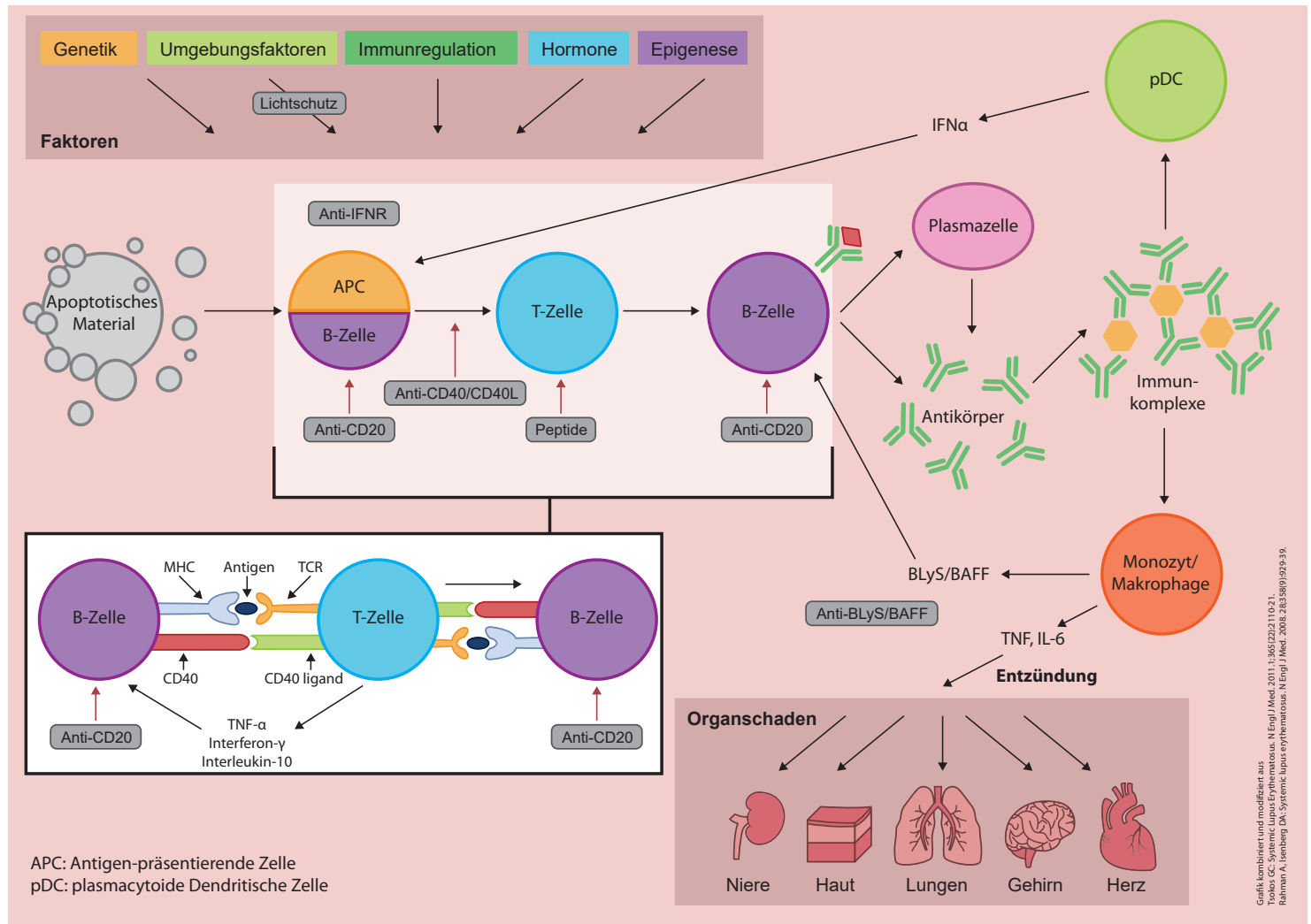


Fachtagung zur Medizin der Zukunft

\section{Chronisch-entzündliche Erkrankungen maßgeschneidert behandeln}

\section{Individuelle Unterschiede erkennen und therapeutisch nutzen}

Chronische Entzündungserkrankungen wie Morbus Crohn, Schuppenflechte, Diabetes oder Rheuma unterscheiden sich nicht nur untereinander, sondern auch von Mensch zu Mensch. Bei diesen Unterschieden setzt die Präzisionsmedizin an: Sie will die individuellen Unterschiede zwischen einzelnen Betroffenen erkennen und diagnostisch und therapeutisch nutzbar machen. Ziel ist es u.a. messbare biologische Marker zu finden, die Voraussagen erlauben, welche maßgeschneiderten Therapien für jeden individuellen Fall sinnvoll eingesetzt werden können.

Die Präzisionsmedizin war das zentrale Thema des 7. Internationalen Symposiums «Inflammation Medicine - From Bench to Bedside», zu dem das Exzellenzcluster «Precision Medicine in Chronic Inflammation» (PMI) Mitte Februar 2020 nach Hamburg einlud. Auf der Fachtagung diskutierten rund 250 internationale Wissenschaftlerinnen und Wissenschaftler aus Klinik und Grundlagenforschung die neuesten Fortschritte in der Entzündungsmedizin. Dabei tauschten sich die Experten über den Stand der Forschung zur Genetik von autoinflammatorischen Erkrankungen, zum Mikrobiom und seinem Stellenwert in der Entstehung von chronischen Entzündungsprozessen sowie über den aktuellen Forschungsstand zu häufigen chronisch-entzündlichen Erkrankungen aus. Ein Angebot speziell für junge Forschende waren die Posterpräsentationen sowie ein Workshop zum wissenschaftlichen Publizieren. «Die Nachwuchsförderung ist eine wichtige Mission für uns», betont Mitorganisator Professor Diamant Thaçi, Dermatologe und Leiter des Exzellenzzentrums Entzündungsmedizin am UKSH, Campus Lübeck.

\section{Zielvorgabe ist die Erforschung der Präzisionsmedizin}

«Bei chronischen Entzündungserkrankungen haben wir das Problem, dass ein Medikament bei einigen Erkrankten gut wirkt und bei anderen nicht», erklärt Cluster-Sprecher Professor Stefan Schreiber, Direktor des Instituts für klinische Molekularbiologie (IKMB) der Christian-Albrechts-Universität zu Kiel (CAU) und Direktor der Klinik für Innere Medizin I am UKSH, Campus Kiel. «Aktuell können wir das überwiegend nur durch Ausprobieren herausfinden». Bereits vor Therapiebeginn vorherzusagen, welche Behandlung bei der individuellen Patientin oder dem individuellen Patienten am erfolgreichsten sein wird, sei daher ein wichtiges Ziel der Forschung, so Schreiber weiter.

Gezielt an praktische Ärzte richtete sich das klinische Symposium «Individualisierte Medizin für chronisch entzündliche Erkrankungen in der Pulmologie, Rheumatologie, Dermatologie und Gastroenterologie». Hier gaben internationale und deutsche Expertinnen und Experten Einblicke in die neuesten Therapiemöglichkeiten entzündlicher Erkrankungen und deren Wirkmechanismen. «Es ist selten so, dass eine Entzündung in einem Organ stecken bleibt», begründet Thaçi den interdisziplinären Ansatz. Es gelte in verschiedenen Organen die Triggerfaktoren und Prädiktoren für Erkrankun-

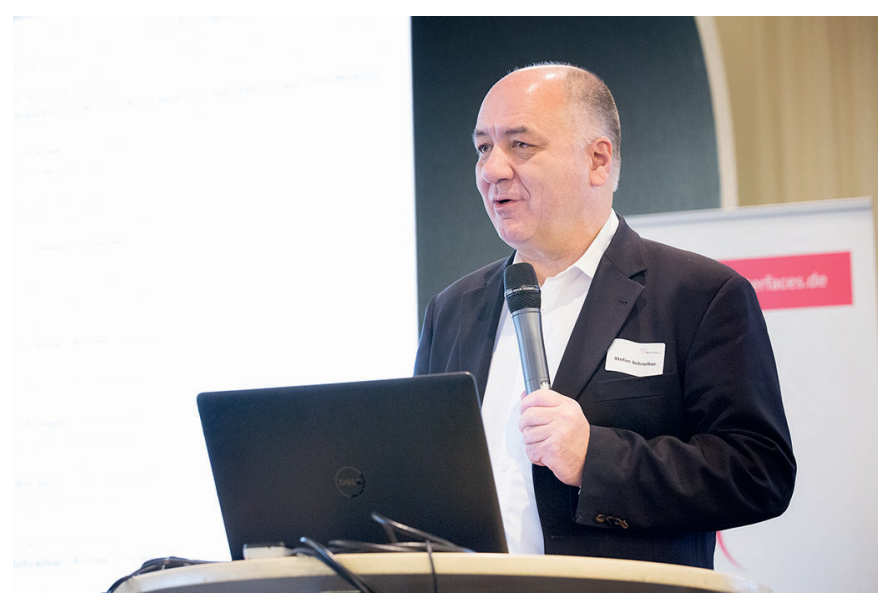

Prof. Stefan Schreiber. @ C. Kloodt, PMI

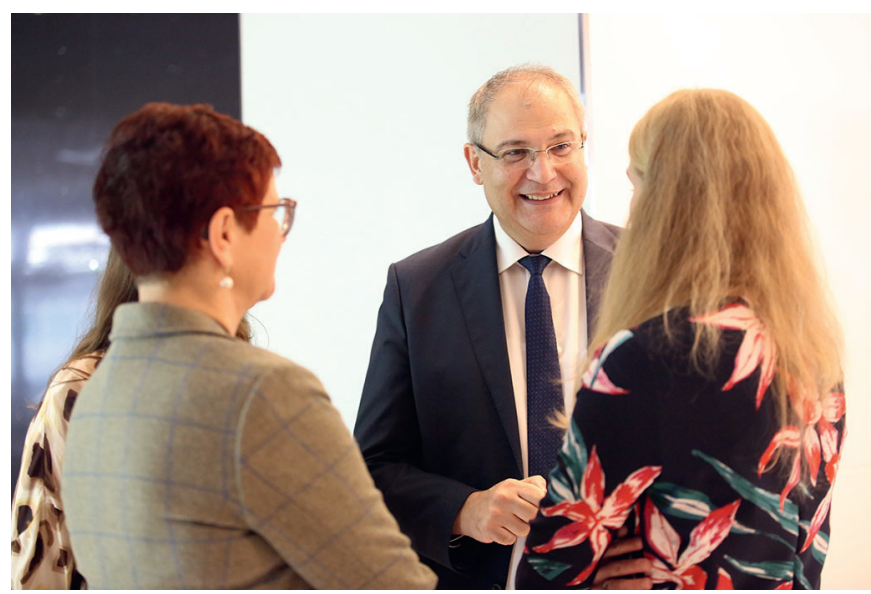

Prof. Diamant Thaçi im Gespräch. @ C C. Kloodt, PMI

gen früher zu erkennen und auf dieser Basis Therapien zu entwickeln, die dem Patienten mehr Nutzen und weniger Risiken bei der Behandlung bringen.

Noch gehört die Präzisionsmedizin zwar nicht zur Standardbehandlung, doch die Forschung nimmt hier Fahrt auf. Thaçi sieht in ihr viel Potential: «Für die Behandlung von chronischen Entzündungen sind bereits einige neuartige Medikamente auf den Markt gekommen, mit denen wir die Behandlung noch präziser als bisher an den zugrundeliegenden molekularen Mechanismen der individuellen Betroffenen ausrichten können.»

Tagungsbericht zum 7. Internationalen Symposium «Inflammation Medicine - From Bench to Bedside» vom 17.-18. Februar 2020 in Hamburg.

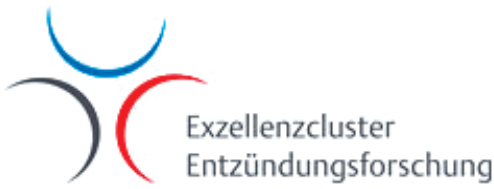




\section{Weiterbildung \\ Fachübergreifende Sekundäranalyse von Daten aus Befragungen zur Arbeits- und Weiterbildungs- situation junger Ärztinnen und Ärzte in Deutschland}

Das Bündnis Junge Ärzte (BJÄ), in dem viele Nachwuchsgruppen verschiedenster Fachbereiche organisiert sind, nutzte seine Reichweite, um den aktuellen und fachübergreifenden Status Quo der Weiterbildungs- und Arbeitsbedingungen junger Ärztinnen und Ärzte in Deutschland zu analysieren. Gerade Änderungen im Gesundheitssystem in den letzten Jahren hatten starken Einfluss auf den Arbeitsalltag der jungen Ärzteschaft. Für das Projekt des BJÄ wurden sich überschneidende Daten aus 6 Einzelbefragungen gemeinsam in Form einer Sekundäranalyse ausgewertet. Insgesamt gingen in die Analyse 4041 komplette Datensätze ein. Inhalt aller 6 Befragungen war neben wichtigen Themenfeldern wie den Arbeitsbedingungen im Berufsalltag und der Weiterbildung sowie der Vereinbarkeit von Beruf und Familie auch das Modell beruflicher Gratifikationskrisen zur Beurteilung psychosozialer Arbeitsbelastung.

Im Folgenden soll ein Überblick über zentrale Ergebnisse der Analyse gegeben werden: Es wurde deutlich, dass ein hoher Anteil der Arbeitszeit durch nicht unmittelbar ärztliche Aufgaben eingenommen wird. Durchschnittlich wurde allein ein Viertel der Arbeitszeit (25 $\pm 15 \%$; Mittelwert \pm Standardabweichung) auf patientenferne Tätigkeiten, wie Befunde und Termine organisieren oder Diagnosen kodieren, verwendet. Die Arbeit direkt am Patienten nahm durchschnittlich 44 $\pm 19 \%$ der Arbeitszeit ein, 32 $\pm 13 \%$ wurden auf patientenbezogene Tätigkeiten wie Fallbesprechungen und Schreiben von Arztbriefen verwendet. Die zunehmende Bürokratisierung und Reglementierung der Medizin in den letzten Jahren ist am ehesten als Grund dieses großen Anteils an nicht unmittelbar ärztlichen Aufgaben zu sehen. Die Mehrheit der Befragten sahen diese vielschichtigen Veränderungen im ärztlichen Arbeitsumfeld deutlich kritisch: Auf die Frage, ob die Qualität der Patientenversorgung dadurch gefährdet sei, antworteten 47\% der Befragten mit «Ja, eher schon» und $40 \%$ mit «Ja, sehr deutlich». Die Befragten wünschten mehrheitlich eine Reduktion der Arbeitsverdichtung z.B. durch eine Entlastung bei organisatorischen Aufgaben und der Dokumentation. Obwohl die aktuellen Arbeitsbedingungen kritisch bewertet wurden, war die allgemeine Zufriedenheit der Befragten mit ihrem Berufsalltag relativ hoch: 10\% der Teilnehmer gaben an «sehr zufrieden», 36\% «eher zufrieden», 28\% «unentschieden», 20\% «eher unzufrieden» und nur 6\% «sehr unzufrieden» zu sein. Nach Ansicht der Autoren lässt sich diese Diskrepanz am ehesten durch eine tiefgehende Identifikation der Ärzteschaft mit ihrem Beruf erklären.

Die psychosoziale Arbeitsbelastung war bei den Teilnehmern der Studie mit einer ER-Ratio von 1,8 stark ausgeprägt. Die ER-Ratio ergibt sich aus 16 Items und setzt Belastungsfaktoren (engl. effort) und Belohnungsfaktoren (engl. reward) ins Verhältnis. Ein Wert um 1 deutet auf ein Gleichgewicht beider Seiten hin. Durchschnittliche Werte aus der deutschen Erwerbsbevölkerung liegen dagegen um 0,5 bis 0,6 (also eine deutliche Verschiebung des Quotienten Richtung Belohnungsfaktoren). Instrumente einer guten Weiterbildung wie Weiterbildungsverträge, feste Curricula mit geplanten Rotationen und regelmäßige Weiterbildungsgespräche waren mit einer geringeren psychosozialen Arbeitsbelastung assoziiert.

Als Fazit der Untersuchung stellen die Autoren fest, dass viele junge Ärztinnen und Ärzte die aktuellen Arbeits- und Weiterbildungsbe- dingungen als belastend empfinden, was nicht nur Konsequenzen für sie selbst, sondern auch für ihre Patienten haben kann. Es sollte im Interesse aller an der Organisation des Gesundheitswesens beteiligten Partner liegen, ein Umfeld herzustellen, in dem Ärztinnen und Ärzte dauerhaft gesund und effektiv arbeiten können. Nur so wird die wertvolle Ressource «Arzt» bestmöglich in der aktiven Patientenversorgung gehalten und eine hochwertige Patientenversorgung auch zukünftig gewährleistet.

Diesem Beitrag liegt die Studie Joachim SC, Bitzinger D, Arnold H, et al.: Die Arbeits- und Weiterbildungssituation junger Ärztinnen und Ärzte in Deutschland - eine zusammenfassende Analyse von Befragungsergebnissen aus sechs Fachrichtungen. Das Gesundheitswesen 2019;DOl:10.1055/a-0945-9387 zugrunde.

Aus dem Bündnis Junge Ärzte (BJÄ) waren folgende Nachwuchsgruppen an der Analyse beteiligt:

- Junge Internisten der Deutschen Gesellschaft für Innere Medizin e.V. (DGIM) und das Junge Forum des Berufsverbandes Deutscher Internisten e.V. (BDI)

- Wissenschaftlicher Nachwuchs (WAKWiN) und Junge Anästhesie der Deutschen Gesellschaft für Anästhesiologie und Intensivmedizin e.V. (DGAI) sowie der youngBDA des Berufsverbandes Deutscher Anästhesisten e.V. (BDA)

- German Society of Residents in Urology e.V. (GeSRU)

- Forum Junge Gynäkologie der Deutschen Gesellschaft für Gynäkologie und Geburtshilfe e.V. (DGGG)

- Arbeitsgemeinschaft Young DOG der Deutschen Ophthalmologischen Gesellschaft e.V. (DOG)

- Forum Junge Radiologie der Deutschen Röntgengesellschaft e.V. (DRG)
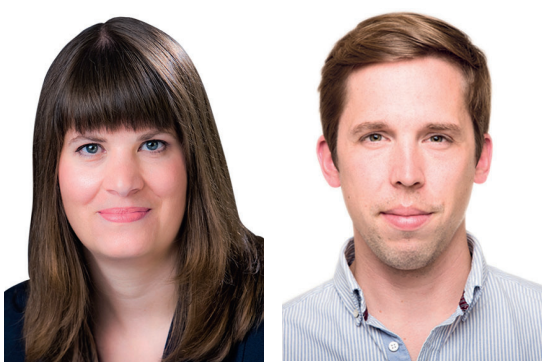

Die beiden ehemaligen Campus-Koordinatoren Prof. Dr. Stephanie Joachim (OphthalmoCampus) und Dr. Matthias Raspe (PneumoCampus) waren an der Studie beteiligt

Das Bündnis Junge Ärzte (BJÄ)

Das BJÄ ist der breite Zusammenschluss ärztlicher Nachwuchsgruppen in Deutschland. Das BJÄ vertritt die Belange junger Ärztinnen und Ärzte im Gesundheitswesen und bringt sich aktiv in aktuelle Themen ein - zum Wohle einer hochwertigen Patientenversorgung und guter ärztlicher Arbeits- und Weiterbildungsbedingungen.

Zur Internetseite der BJÄ: www.buendnisjungeaerzte.org.

Kontakt: Prof. Dr. Stephanie Joachim, Experimental Eye Research Institute, Universitäts-Augenklinik, Ruhr-Universität Bochum, In der Schornau 23-25, 44892 Bochum; Dr. Matthias Raspe, Medizinische Klinik mit Schwerpunkt Infektiologie und Pneumologie, Charité - Universitätsmedizin Berlin, Augustenburger Platz 1, 13353 Berlin, matthias.raspe@charite.de 